\title{
COMPREHENSIVE REVIEW ON IOT BASED HEALTH ARCHITECTURES AND FOG, CLOUD COMPUTING
}

\author{
Sonali Mahajan \\ Electronics and Telecommunication Engineering Department, \\ Deogiri Institute of Engineering and Management Studies, Aurangabad, India. \\ A.M. Birajdar \\ Assistant Professor, Electronics and Telecommunication Engineering Department, \\ Deogiri Institute of Engineering and Management Studies, Aurangabad, India.
}

\begin{abstract}
Traditional health care systems are replaced by use of high precision sensors and IOT enabled medical devices. The m-health system is subset of E-health system .It has gained more popularity over E-Health system due to extensive use of smart phone. Both systems are useful in measurement of physiological as well as chronic health parameters. Microcontroller system processes patient's real time health data and send over cloud or fog. Cloud computing facilitates rapid on demand access to shared pool of virtual computing resources, servers, networks. Fog computing can be considered as extension of cloud computing as it provides low latency, low bandwidth with increased level of data security and privacy. Data breach is major issue in health care system that can be solved using special privacy acts, and special algorithms. In this paper, we are doing comprehensive analysis of IOT based m-health system and E-health system, cloud, fog computing as well as data security issues
\end{abstract}

Key words: E-health, IOT, m-Health, NFC, RFID.

Cite this Article: Sonali Mahajan and A.M. Birajdar, Comprehensive Review on IoT Based Health Architectures and Fog, Cloud Computing, International Journal of Advanced Research in Engineering and Technology, 10 (3), 2019, pp 112-117.

http://iaeme.com/Home/issue/IJARET?Volume=10\&Issue=3

\section{INTRODUCTION}

Due to modernization and technological advancement, our life style patterns changes and it has negative effects on health physically, psychologically, and socially. High intake of fast foods, Lack of physical activity, smoking and alcohol intake, burden of work commitments, people are suffering with hypertension, obesity, Diabetes, Heart disease, Stroke, cancer .Use of Artificial intelligence and IOT in health care is now booming nowadays. IOT facilitates remote health monitoring. IOT in healthcare system is an architectural framework of interconnected sensor network which acquires, exchanges patient's real time health data. GSM -GPS based IOT system not only tracks the record of patient's health condition but also tracks position, 
location of patient in emergency condition [1].Nowadays several wearable activity trackers, fit bit devices are used to monitor real time heart rate, temperature, ECG parameters. Wearable activity trackers are useful in protecting women against sexual harassment and child kidnapping [2]. MEMS devices (micro-electromechanical system) are small size with cheap cost, low powered accurate sensors usually used in accelerometers, inclinometer, gyroscopes, and magnetometer. Accelerometer specially measures static and dynamic acceleration in $\mathrm{x}, \mathrm{y}, \mathrm{z}$ axes used mainly for fall detection of old age patient [3].

In this paper we focus on various IOT based health architectures, classifying sensor modules according to measurement of physiological, chronic health parameters, fog and cloud computing structures their advantages as well as disadvantages and Challenges in maintaining data security. The paper is organized as follows: In section II literature survey. In section III, we outline various IOT based health care architecture. In Section IV, we classify health parameters and related sensor modules .In section $\mathrm{V}$ we discuss about fog and Cloud computing structure. At last section we highlight data security challenges and security act.

\section{LITERETURE REVIEW}

In RFID based Healthcare system proposed in [4 ], physiological health parameters like body temperature, heart rate, ECG is measured using sensor network and patient tracking and monitoring done by RFID tag with reader unit. Measured sensor data is processed by using Arduino-Uno and displayed on LCD and then send to doctors through email using SMTP protocol interfaced with LabVIEW. The LabVIEW is high speed graphical programming language that permits operator to process collected data using complex data algorithm without any knowledge of data acquisition system .Use RFID technology with LabVIEW interfacing increases operational efficiency and patients are identified, tracked in real time. RFID tags used in RFID system can active or passive. Active tag powered through embedded circuitry and passive tag powered through RF signals from reader.

In 2GRFID based healthcare system, tags stores health information in form of mobile codes [5]. RFID reader receives mobile codes and interprets it. Doctor can retrieves patents medical history from RFID reader and writes prescription, diagnosis in form of mobile codes into RFID tag. The whole system built RFID tag, RFID reader with wireless sensor network, mobile phone. Zig-bee wireless communication is used to send real time sensor data on to android phone. As compared to $1 \mathrm{G}$ System, $2 \mathrm{G}$ system is flexible, scalable but costly when memory of RFID tags is increased. RFID system provides real time health tracking with increased asset utilization, reduce medical error, and reduce capital cost.

The NFC based healthcare monitoring system uses wireless sensor network comprised of ECG sensor, Temperature, pulse rate sensor and NFC read/write device with NFC tag, database [6]. NFC based system used for short range two way communications. NFC tag holds information patents name, patient health data, allergies, vaccination, and chronic illness. Authorized doctor can extract information about patient by tapping NFC tag or accessing database using login ID and password. Database has to be updated for maintaining Electronic health Record (EHR). NFC tag helps in maintaining health records.

Bluetooth/BLE based chronic Care model is designed for remote monitoring of chronic patients [7]. The wireless sensor network in system acquires biological data such as ECG oxygen saturation, calories intake, heart rate, and provides connectivity to local gateway. The local gateway provides local storage it contains all computational and communication resources. Further secured sensor data transmission from gateway is possible by use of XML and standardHL7CDA format. Repository is implemented via relational database and it provides long term storage for maintaining EHR and web server Saas application module helps medical staff to manage and update treatment plan for each patient. Module helps in measuring past and 
current vital health parameters. Web services and web application both uses HTTPs protocol to enforce authenticity, integrity and confidentiality of data communication. Bluetooth/ BLE communication provides low powered short range communication. BLE (Bluetooth low energy) has high efficiency in terms of number of bytes to be sent in terms of joules. XBEE based heart rate monitoring system for sport training proposed by I.R.Thenmozhiselvan [8].In the system heart rate detected by sensor is stored in PIC16F877 microcontroller and transferred to Xbee transmitter then data is transmitted using the Xbee wireless link. On the receiving end, another Xbee module receives data and passing it onto another RS-232 serial port to which PC is connected. Data regarding heart rate is continuously displayed in PC. A database is created using Visual Basic. The database includes name of athlete, heart beat range, time and date. System is used to measure Athlete's performance

GSM/GPS based health monitoring system is proposed in [1].The liliypad temperature sensor, pulse sensor, GSM/GPS module and Arduino Uno integrated together for tracking health status of heart patient. Whenever sensor measured values are not as per configured threshold values, SMS alert send via GSM module. Restful software used has three- tire architecture. Front end is HTML web page for viewing real time sensor data, middle tire is server developed using PHP programming and backend tire hosts MySQL database. Secured health monitoring using GSM module and ESP8266WIFI module is proposed in [9].GSM MODEM operates by accepting SIM card into the subscribed mobile operator.GSM modem and pc/laptop can communicate over mobile network. The microcontroller PIC18F46K22 processes sensor values from tempreture,spo2,ESP8266 WIFI module encrypts sensor values using AES128 standard. By logging to HTML web page users/doctors can visualize patient health data and GSM sends alert message to doctor in emergency situation.

\section{OVERVIEW OF IOT ARCHITECTURE IN HEALTHCARE SYSTEM}

\subsection{E- Health architecture}

E-health system is an integration of electronics communication and IOT technology for real time health monitoring of patient. The m-health system is subset of E-health system. E-health system involves patient health data management, appointment scheduling, and maintenance of EMR/EHR. E-Health monitoring application can be used for chronic patients suffering from diseases like asthma, blood sugar [10]. E-Health system architecture consists of data collection, data integration, fog computing layer and cloud computing layer [11]. Data collection is done by wearable sensors. Data integration is achieved by using microcontroller which interfaced with communication standards and sensor network. Fog layer holds time sensitive data, it involves preprocessing of data. Cloud layer have large secured storage of medical reports, medical prescription, patients historical data.

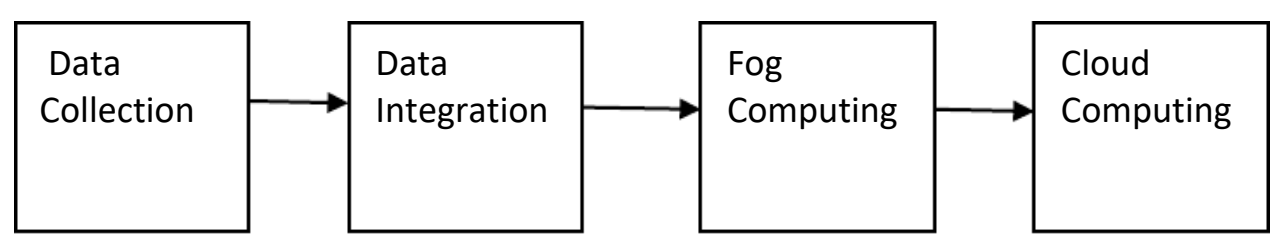

Figure 2 E-Health System 


\section{2. m-Health System}

$\mathrm{m}$ - Health stands for mobile health. It is subset of E-health system-health system which involves use of mobile phone's core functionalities and applications such as SMS service, voice messaging, General Packet Radio Service (GPRS), Global Positioning System(GPS),Bluetooth technology. The m-health system has three level architecture namely, data Collection layer, data storage layer, data processing layer [12].

\section{CLOUD AND FOG COMPUTING FOR HEALTH CARE SYSTEM}

Data acquired from sensor not lasts for longer time .It is necessary to process and store for further usage. IOT facilitates use of fog and cloud computing for processing real time sensor data

\subsection{Fog computing}

Fog or Edge computing is an expansion of cloud computing [13]. Fog computing is designed to handle volume, variety, and velocity of IOT Data where requirement of reduced latency time, saving of bandwidth, security and reliability are essential parameters [14]. Fog node placed at the edge of local network much closed to IOT devices for acquiring time sensitive data; they look for problem issues and prevent them by sending commands through actuators. Fog computing is help in faster response time, low bandwidth with higher level of security and privacy. Fog computing uses simple analytics and visualization

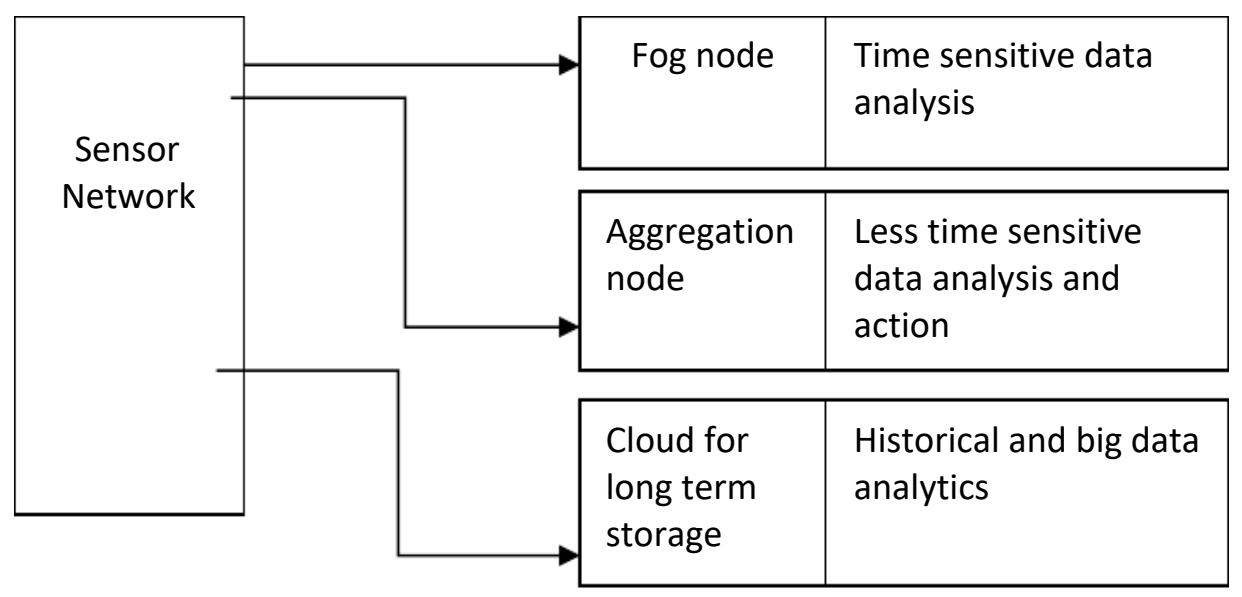

Figure 2 Fog Structure for sensor data computing

Fog node is closest to sensor network for acquiring time sensitive data, it checks for any dangerous problem. Aggregation node hold less time sensitive data, hold it for minutes can be used for data analytics and taking corresponding action. Cloud receives data from various fog nodes and uses it for big data analysis.

\subsection{Cloud Computing}

It has flexible and dynamic architecture for medical application and healthcare system. Some of features its includes on demand resource allocation to specific need from entire network, scalable architecture that promotes expansion of functional capabilities well as computational power, resource pooling by multiple users from service provider for accessing its computing facilities and broad network access[15]. Cloud computing can have3 leveled structure that includes Storage, analytics and visualization. . Cloud storage can be public, private, hybrid. Public cloud is cost effective, open to public and managed by service provider and data access 
and data breach problem arises with it. Private cloud is costly managed by single organization it controls manages sensitive patients data within that organization. Cloud Analytics is service model where data analysis is performed on public or private cloud. It can be Iaas, Paas, Saas[16].The Iaas means infrastructure as service in which organization provided with infrastructure including networks, servers, and storage computing services . The paas means Platform as a service infrastructure, where service providers provides a platform or software for users and user develop, modify and maintain the application. The Saas means Software as a service which allows user for use cloud based application over the internet and user can have remote access to this application using web browser.

\section{DATA SECURITY CHALLENGES AND PRIVACY ISSUES}

\subsection{Data integrity and Authentication}

For improving data security and privacy, two encryption algorithms are used namely AES and RSA based PHE. The AES (advanced encryption standard) is used for encrypting patient's health data for cloud storage [16]. RSA based PHE (partial homomorhpic encryption) technique helps to encrypt AES encryption keys when patient's health data storage and management is done by third party.

\subsection{Data security act}

Data breach creates significant harm to patient health data. Both cloud providing venders and cloud user wants to avoid this data loss. In such conditions special data security acts plays important role.Privacy and security of health data are important factors in health management systems. HIPPA (Health Insurance Portability and Accountability Act) safeguards patients privacy and patients health data. HIPPA implementation requires cloud computing vender, third party to sign business associate agreement to access health data from health oragnisation.[15].

\section{CONCLUSION}

The paper provides a comprehensive survey of various IOT based health systems, health parameters, cloud and fog computing structure and data security issues. M-health system is subset of E-health system gained more popularity as use of low cost portable smart phones. GPS/GSM in smart phones helps to track location of patients and sending SMS alerts in emergency. Remote health monitoring and providing telemedicine avoids frequent appointments to doctors by use of E-health and M-health system. Cloud computing provides long term data storage and helps in maintaining EHR/EMR whereas fog computing stores transient data , uses simple analytics and visualization. It provides low latency and low bandwidth.

\section{REFERENCES}

[1] KantanAziz," Smart Real Time Healthcare and Tracking System using GSM/GPS Technologies", IEEE International Conference on Big Data and Smart City, 2016.

[2] T Bhanupriya, " Activity Tracker Wrist Band for Children Monitoring using IOT”, International Journal on Recent and Innovation Trends in Computing and Communication, vol5,Issue11, November 2017,pages 52-57.

[3] Sadik Kamel Gharghan," Accurate fall Detection and Localization for Elderly people Based on Neural Network and Energy-Efficient Wireless Sensor network", Energies, 2018, DOI: $10.3390 \mid$ en 11112866.

[4] Dr. R. M. S Parvathi, M.Aisswaryya," RFID based Patient Monitoring and Mobile Alert System in Healthcare Organization , National Conference on networks, Intelligence and Computing System( IJIRST), March 2017. 
[5] Min Chin," A 2G- RFID BASED HEALTHCARE SYSTEM", IEEE Wireless Communications, February 2010.

[6] Supriya A,S.Ramgopal, “ Near Field Communication Based System for health Monitoring ,IEEE international Conference On Recent Trends in Electronics information and Communication Technology , 19 may 2017, India.

[7] Massimiliano Donati," A Telemedicine Service Platform Exploiting BT/ BLE wearble Sensors for Remote Monitoring of chronic Patients “, IEEE International Conference on Modern Circuits and System technologies, 2018.

[8] R.Thenmozhiselvan, “ XBEE BASED HEART RATE MONITORING IN SPORT TRAINING “, International journal of Power Control and Computation (IJPCSC),VOL7,2015, Singapore.

[9] Bhoomika B.K., "Secured Smart Healthcare Monitoring System Based on IOT", International Journal on Recent and Innovation Trends in Computing and Communications, vol3, Issue7, July 2015.

[10] Radu Ciorap, "E-Health System for Monitoring of Chronic Diseases", Research Gate Conference paper, January 2010.

[11] Kayo Monterio, "Develpoing an e-health system based on IOT, fog and cloud computing", IEEE/ACM International Conference on Utility and Cloud Computing.

[12] Niharika Kumar, "IOT Architecture and System Design for Healthcare Systems", IEEE2017.

[13] MirjanaMaksimovic," IMPLEMENTATION OF FOG COMPUTING IN IOT”, Journal of Information Technology and Applications (JITA),2017.

[14] Wang, Lidong \& Alexander, Cheryl. Medical Applications and Healthcare Based on Cloud Computing. International Journal of Cloud Computing and Services Science (IJ-CLOSER). (2013). 2. 10.11591/closer. V 2i4.3452.

[15] Mohammed Faez Al-Jaberi, "Data Integrity and Privacy Model in Cloud Computing”, IEEE International Symposium on Biometric and Security Technologies (ISBAST),2014.

[16] M N Rajaprabha, P Jayalakshmi, R Vijay Anand and N Asha, Iot Based Smart Garbage Collector for Smart Cities, International Journal of Civil Engineering and Technology, 9(12), 2018, pp. 435-439

[17] Dr. Samir Mohammed Chassib, Dr. Sa'ad Fahad Resan, Mohammad Salah Gejan, Murtadha Jabar Salih, Ali Mohsen Hasan, Developing Sustainable Lightweight Bubbled Ferrocement Slab Using Enhancing Cementitious Agents, International Journal of Civil Engineering and Technology (IJCIET) 9(11), 2018, pp. 749-765.

[18] T. Aravinda Babu and K.S.R.S. Jyothsna, IOT Based Smart Vehicle and Smart Parking System: International Journal of Electrical Engineering \& Technology, 9(3), 2018, pp. 121-136.

[19] Sharmila Nath, Jayanta Kumar Nath and Kanak Chandra Sarma, IoT Based System for Continuous Measurement and Monitoring of Temperature, Soil Moisture and Relative Humidity. International Journal of Electrical Engineering \& Technology, 9(3), 2018, pp. $106-$ 113.

[20] IOT Based Toll Collection System Using Image Processing, Malvik Patel, Bharavi Joshi, Kajal Bhagat and Hetakshi Desai and Jekishan K. Parmara. International Journal of Computer Engineering \& Technology, 9(3), 2018, pp. 132- 139.

[21] Mr. N. Sampathraja, Dr. L. Ashok Kumar, Mr. K. Vishnu Murthy, Ms. V. Kirubalakshmi and Ms. C. Muthumaniyarasi, Iot Based Underground Cable Fault Detector, International Journal of Mechanical Engineering and Technology 8(8), 2017, pp. 1299-1309. 